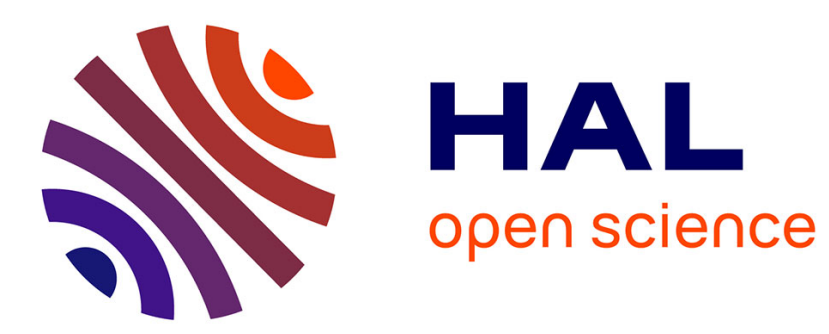

\title{
Ecologie et intérêt sylvicole de divers Sorbus en France
}

Nicolas Drapier

\section{To cite this version:}

Nicolas Drapier. Ecologie et intérêt sylvicole de divers Sorbus en France. Revue forestière française, 1993, 45 (3), pp.345-354. 10.4267/2042/26431 . hal-03444076

\section{HAL Id: hal-03444076 https://hal.science/hal-03444076}

Submitted on 23 Nov 2021

HAL is a multi-disciplinary open access archive for the deposit and dissemination of scientific research documents, whether they are published or not. The documents may come from teaching and research institutions in France or abroad, or from public or private research centers.
L'archive ouverte pluridisciplinaire HAL, est destinée au dépôt et à la diffusion de documents scientifiques de niveau recherche, publiés ou non, émanant des établissements d'enseignement et de recherche français ou étrangers, des laboratoires publics ou privés. 


\title{
ÉCOLOGIE ET INTÉRÊT SYLVICOLE DE DIVERS SORBUS EN FRANCE
}

\author{
N. DRAPIER
}

L'Alisier torminal n'est pas le seul Sorbus méritant d'être réservé ou planté en forêt. À divers degrés, c'est aussi le cas du Cormier (Sorbus domestica), de l'Alisier blanc (Sorbus aria), du Sorbier des Oiseleurs (Sorbus aucuparia), de l'Alisier de Fontainebleau (Sorbus latifolia) et des hybrides d'Alisiers blanc et torminal (Sorbus $x$ confusa). De plus, les possibilités d'emploi de ces essences présentent d'intéressantes complémentarités.

À l'origine, il nous est apparu intéressant de reconsidérer les connaissances existantes sur les autres Sorbus à la lumière des acquis de notre étude écologique de l'Alisier torminal. II devait s'avérer que cette approche conjointe de plusieurs espèces voisines pouvait être fructueuse pour la compréhension de leurs comportements écologiques et de leurs possibilités complémentaires de valorisation.

Cependant, les considérations qui suivent résultent uniquement d'observations empiriques et d'études bibliographiques, sans l'appui de prospections et analyses rigoureuses. C'est pourquoi nous nous restreindrons à des considérations générales et prudentes qui, malgré cela, ne sont peut-être pas à l'abri de défauts que nous avons précédemment cités à propos de certaines publications traitant de l'Alisier torminal.

\section{LE CORMIER}

\section{Écologie}

Des trois principaux autres Sorbus français, le Cormier est celui dont l'écologie se rapproche le plus de celle de l'Alisier torminal.

Jusqu'à maintenant, cette espèce a surtout été considérée comme une mésoxérophile, compagne souvent de l'Alisier blanc (encore que, comme pour l'Alisier torminal, l'opinion que s'en soient faite bien des auteurs ait surtout été fonction de la région de leurs observations). L'amplitude écologique du Cormier est en fait beaucoup plus large, ce qui pourra avoir d'intéressantes applications pratiques.

Comme l'Alisier blanc, le Cormier se rencontre régulièrement (mais toujours très disséminé) dans des milieux secs, surtout sur calcaire.

Comme l'Alisier torminal, on le trouve dans des forêts acidiphiles plus ou moins dégradées du Bassin parisien (entre autres), où il manifeste une meilleure adaptation que l'Alisier aux situations les plus sèches (par exemple sur sols lessivés à horizon éluvial grossier et filtrant ou bien limoneux et tassé, à sécheresse estivale aggravée par le climat). 


\section{N. DRAPIER}

II serait cependant hâtif de voir dans ces exemples l'expression d'une tendance subméditerranéenne beaucoup plus affirmée chez le Cormier que chez l'Alisier torminal. Car si le Cormier est relativement plus fréquent dans le Sud, il peut suffire pour cela que certains facteurs stationnels lui soient moins défavorables qu'ailleurs, en particulier que la concurrence soit inférieure - ce que peuvent offrir des peuplements forestiers bas et peu denses, improductifs et souvent dégradés, comme il en existe beaucoup dans ces régions. Si donc le Cormier a été plus favorisé dans le Sud que ne l'a été l'Alisier, il faudra sans doute des plantations comparatives, les plaçant à égalité au départ, pour savoir ce qu'il en est vraiment des potentialités de ces deux essences.

Le Cormier atteint en montagne une altitude supérieure à l'Alisier: $1400 \mathrm{~m}$ (d'après la Flore forestière française) au lieu de 1000 ; c'est par contre inférieur à l'Alisier blanc (1700 m), dont le Cormier n'a pas la tendance continentale. Il est possible que sa rustícité lui permette de monter à la faveur d'adrets ou autres situations sèches et ensoleillées (même si pas particulièrement chaudes: en montagne, la lumière peut ètre une contrainte en soi); cette remarque vaudrait également pour l'Alisier blanc.

De même qu'il apparaît que la tendance subméditerranéenne est à aussi large amplitude que celle de l'Alisier torminal, il s'avère aussi que son apparente mésoxérophilie n'est encore que l'expression d'une compétitivité plus faible que celle des autres grands Sorbus.

Mais cette sensibilité du Cormier à la concurrence est particulière. Par son développement et sa croissance, il pourrait être le plus forestier ; il est en effet le plus apte à différencier un axe principal bien individualisé, à bien croître avant de développer son houppier; il est moins souvent affligé de fourches, et capable peut-être d'atteindre une plus grande taille que l'Alisier torminal ; enfin, il est aussi plus longévif. Mais, outre son héliophilie qui est certaine, le principal inconvénient du Cormier sur le plan dynamique est sa régénération: il drageonne peu, se trouve donc davantage tributaire des semis; aussi la concurrence s'exerce-t-elle dès le début sur des plants peu nombreux et, pour les francs-pieds, initialement moins vigoureux que des drageons.

La très large amplitude écologique du Cormier se trouve encore illustrée par sa présence et ses bons résultats dans deux types de milieux oủ il côtoie le torminal.

Nous avons déjà cité la présence régulière du Cormier dans les chênaies acidiphiles à sylvofaciès favorable d'lle-de-France, du Centre, et jusqu'en Poitou-Charentes. II est le plus fréquent dans des milieux séchards, favorisé par sa résistance à cette contrainte ; c'est ainsi également qu'on le retrouve en Midi-Pyrénées (où il a par ailleurs une large tolérance en ce qui concerne le substrat). Cependant, dans ces milieux acides, les plus beaux sujets sont sur des sols lessivés à engorgement temporaire et régime hydrique contrasté (ce qui les aura avantagés par rapport à la concurrence) plutôt que dans des milieux à contrainte xérique permanente.

Fait plus surprenant, on trouve également le Cormier sur le Plateau lorrain, sur marnes, et il y donne encore de très beaux arbres. Les sols sont dans ce cas des pélosols ou des pélosolspseudogleys. Malgré la tonalité continentale du climat lorrain, le Cormier ne s'y comporte donc pas en calcicole thermophile, contrairement à ce qu'aurait pu faire croire sa présence préférentielle dans les stations les plus sèches des plateaux calcaires. Comme l'Alisier torminal, c'est encore sa résistance aux pédoclimats contrastés qui sert le Cormier dans les forêts du Plateau lorrain, malgré des conditions de concurrence qui ne sont pourtant pas extrêmement favorables dans les chênaies pédonculées - charmaies (de fait, le Cormier y est rare).

Ces deux situations illustrent une qualité du Cormier qui le différencie nettement de l'Alisier blanc : sa tolérance envers des sols lourds, argileux (argile à silex, marnes) ou limoneux (certains sols lessivés).

Ces diverses situations favorables au Cormier ont surtout pour lui l'avantage de ne pas être propices à une présence dynamique du Hêtre. Cependant, comme dans le cas de l'Alisier 
torminal, on trouve quelques rares et remarquables sujets de Cormier dans de hautes futaies régulières de Hêtre, en particulier sur les plateaux calcaires de l'Est. C'est encore l'illustration de potentialités auxquelles on a trop peu donné l'occasion de s'affirmer, et qui sont incontestablement meilleures que celles de milieux calcaires séchards où on envisage trop souvent de confiner le Cormier.

L'impression de très grande plasticité que procure le Cormier peut trouver une part d'explication dans l'usage qui a été fait de cet arbre, car il a été autrefois largement répandu artificiellement pour ses fruits et, déjà, son bois. Mais même s'il n'est alors que subspontané dans certains milieux ou régions - état qu'il aurait au moins eu le "mérite" de maintenir depuis fort longtemps - il faut surtout retenir les conditions écologiques remarquablement variées dans lesquelles on trouve cet arbre.

En résumé, le Cormier est une espèce à part. II est le seul, des Sorbus de la flore française, à ne pas s'hybrider spontanément avec une ou plusieurs autres espèces. II a une très grande amplitude écologique et une très grande rusticité, est excessivement sensible à la concurrence - caractères typiques d'espèce au nomadisme prononcé (on pense surtout au Sorbier des Oiseleurs) - et pourtant, par son architecture et sa longévité, il semble présenter la plus grande évolution vers les dryades. Ces caractères sont en concordance intéressante avec l'étude phyllogénétique et la réorganisation du genre Sorbus qu'ont faite Phipps et al: : le Cormier y est présenté comme espèce unique du genre Cormus, n'ayant que peu d'affinités génétiques avec Aria et Torminalis, et issue du genre Sorbus sensu stricto (Sorbus aucuparia, etc...).

\section{Intérêt sylvicole}

Le Cormier peut donner d'aussi gros et grands arbres que l'Alisier torminal, et son bois est souvent cité comme encore plus beau. En conséquence, cette essence est à conseiller au même titre que l'Alisier pour toutes les situations écologiques intéressantes citées plus haut. Ainsi, en situation mésoxérophile, ou au moins à contrainte xérique saisonnière marquée (cas fréquent en Berry, Poitou, dans le Sud-Ouest), de même dans le Midi, et en basse montagne. À noter, le programme de plantations conservatoires récemment engagé par I'INRA pour les régions du Sud de la France.

Cormier remarquable.

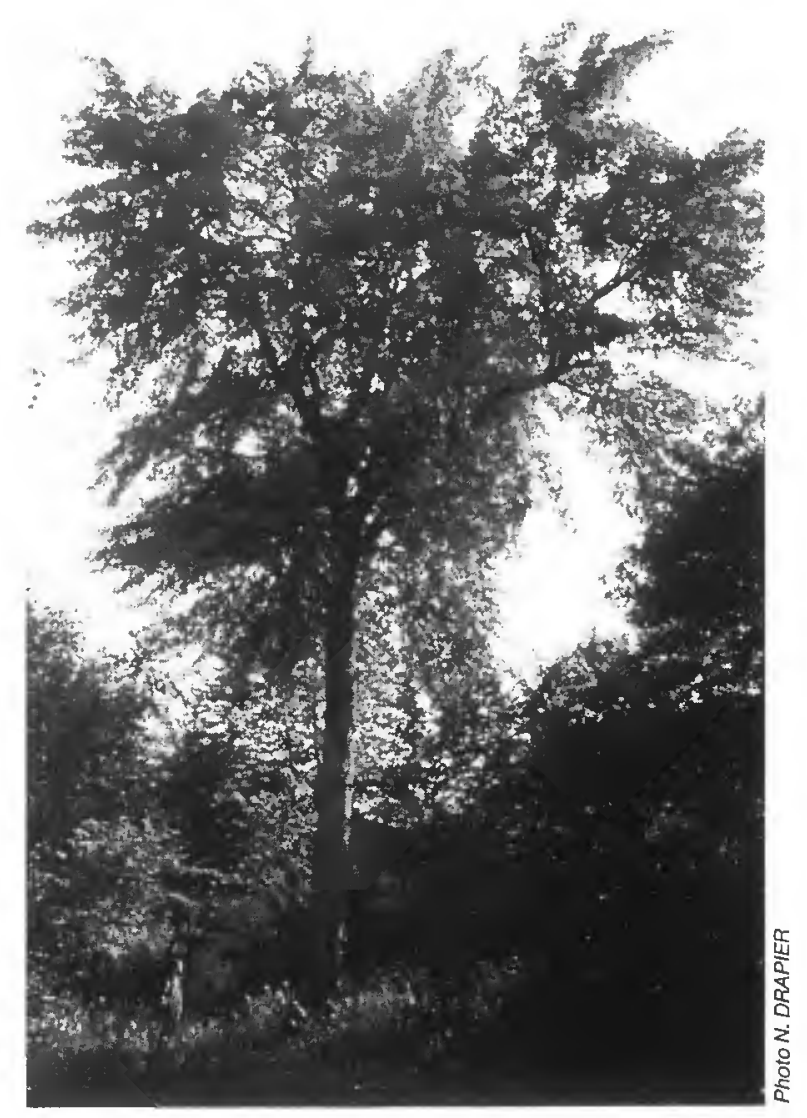




\section{N. DRAPIER}

\section{L'ALISIER BLANC}

\section{Écologie}

Parmi les grandes espèces de Sorbiers existant en France, l'Alisier blanc peut être considéré comme la plus rustique, étant adapté à la plus grande variété de conditions écologiques contraignantes (bien qu'il soit exclu de certaines stations que supportent mieux d'autres Sorbus).

On rencontre fréquemment l'Alisier blanc sur les plateaux calcaires de l'est du Bassin parisien, où il occupe essentiellement des milieux forestiers séchards (mésoxérophiles à xérophiles), à sols fréquemment carbonatés. II y prend souvent le relais de l'Alisier torminal dans les situations les plus sèches.

Comme le torminal, l'Alisier blanc ne dédaigne pas des conditions plus favorables. Mais il se trouve déjà éliminé par la concurrence de peuplements développés sur sols bruns calciques faiblement profonds, particulièrement dès que cette évolution des sols et le climat suffisent au développement du Hêtre et du Charme. De la sorte, l'Alisier blanc se trouve surtout dans des sylvofaciès largement dominés par les Chênes, et plus ou moins dégradés (par surexploitation, non par défaut d'interventions).

En montagne, l'Alisier blanc atteint $1700 \mathrm{~m}$ d'altitude; le Sorbier des Oiseleurs fait mieux, mais sans avoir la même amplitude écologique (on verra la différence de tempérament qu'il y a entre ces deux espèces). Cette résistance de l'Alisier blanc aux contraintes surtout thermiques du milieu montagnard est une forme d'expression de son caractère continental, qui exclut cette espèce de la moitié Ouest de la France, alors que des milieux synécologiquement favorables (landes boisées...) y sont fréquents. Cependant, on remarque que l'Alisier blanc est présent dans les îles britanniques, sous un climat océanique typique; une telle disjonction de l'aire de l'espèce conduit à envisager une évolution différente sur le continent et sur ces îles (de plus, Sorbus aria s'y trouve associé à un complexe d'espèces particulier, avec lesquelles il peut s'hybrider).

II peut être intéressant de noter que l'Alisier blanc, dont l'écologie a des traits communs à la fois avec celles de l'Alisier torminal et du Sorbier des Oiseleurs, s'hybride avec ces deux espèces (et d'autres montagnardes) alors qu'elles ne le tont pas entre elles.

Tout à fait à l'opposé de ses stations sur calcaire, l'Alisier blanc se rencontre également sur des sols très acides. Cet autre cas de contraintes édaphiques prononcées va évidemment de pair avec des peuplements peu évolués ou dégradés, à faible concurrence interspécifique ; de fait, cette situation est souvent en plus montagnarde, de sorte que les contraintes du climat se combinent à celles du sol (en plaine, dans la moitié de la France occupée par l'Alisier blanc, les situations vraiment favorables sont moins fréquentes que sur calcaire: une ambiance forestière déjà affirmée y prévaut en général dans les milieux acides).

II semble cependant, ce qui n'est pas surprenant, que l'Alisier blanc ait un plus petit développement sur sols acides, où les conditions de nutrition minérale sont défavorables et souvent combinées à un pédoclimat sec (à la différence du Sorbier des Oiseleurs, l'Alisier blanc n'est pas fréquent dans des milieux à la fois acides et plus ou moins humides).

Le comportement de l'Alisier blanc vis-à-vis de sols à contrainte d'engorgement même temporaire est illustré par son absence de milieux où l'Alisier torminal a un bon développement (quantitatif et qualitatif) et où - plus significatif - le Cormier est également présent.

Ces milieux sont les forêts sur sols lessivés plus ou moins hydromorphes (particulièrement à mafériau éluvié fin et tassé), et celles sur marnes. Dans ces deux cas, l'humidité est le corollaire de sols lourds, caractéristique qui semble aussi avoir en soi un effet défavorable sur l'Alisier 
blanc (la part des deux facteurs reste à préciser); il est d'ailleurs possible que ceci contribue déjà à limiter son développement sur sols calciques relativement profonds mais assez fortement argileux.

L'appareil végétatif de l'Alisier blanc présente des caractéristiques qui sont en cohérence avec sa rusticité, et sa nature corollaire d'espèce post-pionnière nomade. Á la différence de l'Alisier torminal, il a une capacité prononcée de rejeter de souche et d'émettre des gourmands; ce sont des éléments favorables pour une espèce appelée à se développer dans des milieux contraignants et ouverts, sans la protection qu'un couvert procure face à diverses agressions.

La facilité de rejeter de l'Alisier blanc l'aura en général tait couper avec le taillis dans les taillissous-futaie, lui laissant d'autant moins de chances de bien se développer (à la différence de l'Alisier torminal, souvent balivé). Comme en plus la concurrence l'a confiné à des stations contraignantes, et que ce sont surtout des pratiques sylviculturales intensives (taillis et courtes rotations) qui l'y ont favorisé, les gros sujets d'Alisier blanc sont rares. À défaut, ces mêmes pratiques ont conduit à l'existence de quantités importantes d'Alisier blanc dans de mauvais peuplements qu'ils pourront contribuer à revaloriser.

\section{Intérêt sylvicole}

On considère souvent la qualité bois de l'Alisier blanc comme inférieure à celle de l'Alisier torminal ou du Cormier. II en est pourtant peu différent, et a aussi eu par le passé (et encore un peu de nos jours) des utilisations nobles : mécanique, instruments de mesure. Tout au plus est-il plus fibreux, mais la distinction des bois d'Alisiers blanc et torminal demande de toute façon le recours au microscope.

Le peu d'intérêt qu'on porte maintenant à l'Alisier blanc tient sûrement au très taible marché que cet arbre représente, du fait de la rareté des gros sujets; or, le marché des Sorbus est actuellement presque exclusivement porté par celui des bois de tranchage, exigeant en billes d'assez gros diamètre. Mais on a pu constater, et cela est tout à fait significatif, que des lots d'Alisier n'étaient pas dépréciés à la vente par l'incorporation de blanc, quand l'occasion s'en présentait.

Dans l'avenir, même si l'Alisier blanc reçoit les soins qu'il mérite dans bien des situations, l'intérêt économique de cette valorisation dépendra largement de la façon dont se sera développé le marché des bois d'ébénisterie pour les Sorbus. Ceci est particulièrement vrai pour certains milieux défavorables dans lesquels cette essence sera intéressante, sans pour autant avoir des performances optimales.

D'un strict point de vue sylvicole, l'Alisier blanc mérite surtout que l'on s'intẻresse à lui dans des milieux plus ou moins secs dans lesquels l'Alisier torminal perd de son intérêt, et où luimême dispose souvent d'un important capital de matériel végétatif.

Cependant, comme le torminal, mais plus encore du fait de la clarté habituelle de ces peuplements et de la ramification vigoureuse de l'Alisier blanc, il faudra appliquer à cette essence une sylviculture attentive et semi-intensive dans laquelle les tailles de formations et élagages tiendront une large place.

À cause de ces problèmes de conformation, et de la très forte sensibilité de l'Alisier blanc à la concurrence, il n'y a plus lieu de le préférer au torminal dès que l'on se trouve dans des milieux où celui-ci présente d'au moins aussi bonnes potentialités de croissance. Ceci peut être délicat à apprécier, mais le capital en place sera de toute façon souvent le principal facteur du choix de la conduite sylvicole à tenir (étant donné le genre de peuplements pour lesquels le problème risque de se poser, et la préférence qu'on aura tendance à y accorder à un investissement réduit). 


\section{N. DRAPIER}

\section{LE SORBIER DES OISELEURS}

\section{Écologie}

Des grands Sorbus présents en France, le Sorbier des Oiseleurs est celui qui manifeste la moins large amplitude écologique.

En toute rigueur, il s'avère que c'est encore d'une amplitude synécologique étroite qu'il faudrait parler, dont découle le cantonnement de l'espèce (comme arbre ou grand arbuste) à des milieux à concurrence particulièrement faible. En revanche, l'amplitude autécologique serait plus large qu'il n'y paraît.

En plaine, le Sorbier des Oiseleurs a du moins toutes les apparences d'une vraie espèce acidiphile; mais, en montagne, il est indifférent vis-à-vis du substrat.

En premier lieu, le Sorbier des Oiseleurs est encore une espèce post-pionnière nomade, au nomadisme plus marqué que chez tous les autres grands Sorbus. II est particulièrement rustique, héliophile, supportant les contraintes du climat de montagne jusqu'à $2000 \mathrm{~m}$. La contrepartie en est une faible résistance à la concurrence, surtout que cet arbre est de petite taille (rarement plus de $20 \mathrm{~m}$ ); mais on verra plus loin que le Sorbier des Oiseleurs diffère quand même sur ce point des espèces vues précédemment.

Contrairement aux autres Sorbus, le Sorbier des Oiseleurs a apparemment un véritable optimum de développement (du moins en plaine) pour des milieux acidiphiles, mais la prudence doit rester de rigueur en l'absence d'étude ou de plantations comparatives. II ne s'agit en tout cas pas d'une acidiphilie stricte, comme l'atteste son utilisation dans des conditions très variées en tant qu'espèce ornementale.

upporte en montagne les contraintes chimiques, le Sorbier des Oiseleurs est des milieux formés sur matériau calcaire superficiel, milieux pourtant synécoloables, comme l'atteste la présence fréquente des trois Sorbus vus précédem:e situation, c'est la contrainte xérique qui prévaut. En effet, le Sorbier des oin d'une humidité assez importante, et surtout bien répartie au long de la station. Ce besoin est plus régulièrement couvert sous la fraîcheur du climat me dans des stations à pédoclimat sec (qui tendent à porter une végétation able au Sorbier).

ien, la satisfaction moins fréquente de son besoin d'humidité et de fraîcheur - une compétitivité encore amoindrie, que le Sorbier des Oiseleurs ne soit is des milieux optimaux pour sa nutrition minérale. Elle expliquerait aussi une znante de prime abord, vis-à-vis des couverts fermés qui lui fourniraient en fait que ". Cette adaptation n'en est pas moins remarquable par rapport aux autres i les Sorbiers des Oiseleurs placés dans ces conditions ne sont jamais que des ître peut-on y voir le résultat d'une coévolution avec le Hêtre et le Sapin, dont latiques en font des compagnons habituels du Sorbier.

ce besoin d'humidité et de cette adaptation corollaire à des climats froids (voir tition), le Sorbier des Oiseleurs est l'espèce dont l'aire s'étend en France le plus ns au sud, et qui se trouve le plus au nord de l'Europe. II semble au demeurant ie l'acidiphilie aille de pair avec l'adaptation à l'humidité et au froid, puisque ces ivorisent le développement à grande échelle de sols acides (voir l'ouest de la èrement le secteur phytogéographique armoricain).

èce nomade très rustique du Sorbier des Oiseleurs se retrouve au niveau des son appareil végétatif : forte capacité de se ramifier, de rejeter de souche ou de ment des sujets multicaules, et même de se marcotter (avec tout ce que cela ntages et d'inconvénients pour la sylviculture).
Alors qu'il en $s$ absent en plaine giquement favor ment. Dans cet Oiseleurs a bes période de vége montagnard, mê clairsemée favor

À l'étage colline expliquerait, pa présent que dar tolérance, surpr un "abri climati Sorbus, même s arbustes ; peutles affinités clim Conséquence $d \epsilon$ la carte de répar à l'ouest, le moi assez logique qu deux facteurs fa France, particuli

La qualité d'esp adaptations de donner spontane impliquera d'ava 


\section{Intérêt sylvicole}

Les données sur l'utilisation de cette essence - qualité du bois, milieux favorables, exigences sylviculturales - sont globalement les mêmes que celles de l'Alisier blanc. L'intérêt èconomique du Sorbier des Oiseleurs est cependant fortement limité par la petite taille de ses sujets.

Nèanmoins, il a l'avantage de se prêter à une utilisation à des altitudes supérieures aux autres, et d'y constituer au moins un élément intéressant de diversification de la végétation arborescente. En montagne, le Sorbier des Oiseleurs "fait partie du paysage ", est souvent naturellement abondant, et mérite qu'on essaie de le valoriser comme essence de production de petits bois de sciage. On pourrait l'y essayer aussi bien en milieux assez ouverts (dont des friches) qu'en ambiances plus forestières, où sa relative résistance à la densification des peuplements pourrait faciliter son incorporation en même temps que l'élagage naturel.

\section{L'ALISIER DE FONTAINEBLEAU ET LES HYBRIDES D'ALISIERS BLANC ET TORMINAL}

\section{Écologie}

Certains des caractères morphologiques des hybrides - architecture, émission de gourmands ou de drageons - indiquent que leur nature intermediaire entre Sorbus aria et Sorbus torminalis s'étend à des aspects de la physiologie (croissance et développement). II n'est donc pas surprenant de constater que cette double parentè se retrouve au niveau des affinités écologiques des formes hybridogènes: leur très grande sensibilité à la concurrence et leur tolérance
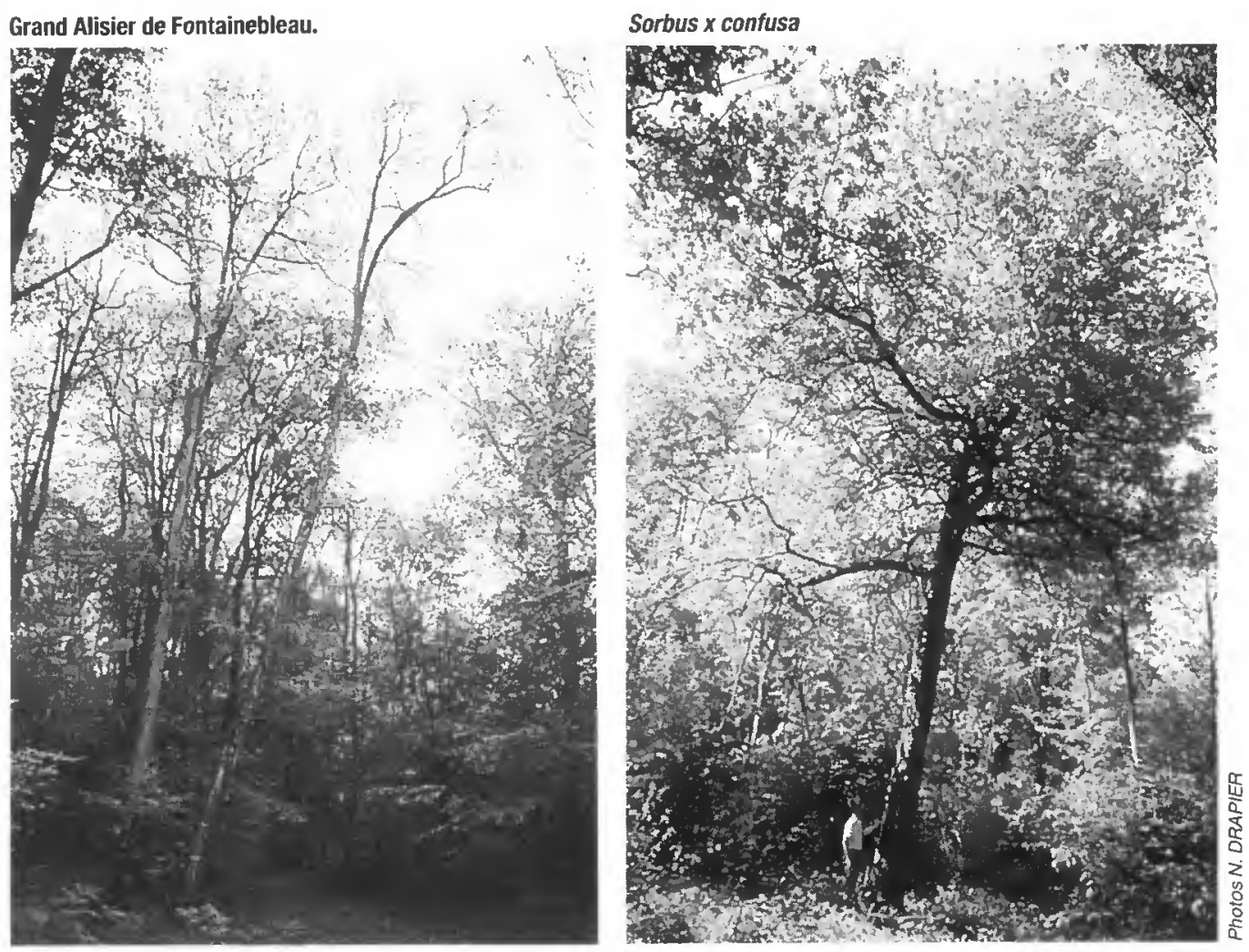


\section{N. DRAPIER}

vis-à-vis de certaines contraintes édapho-climatiques rappellent l'Alisier blanc (en forêt de Fontainebleau, Sorbus latifolia se trouve dans les milieux forestiers les plus acides, les plus secs, les plus calcaires - les plus ouverts aussi), tandis que leur façon de supporter la concurrence les rapproche du torminal - mais dans chaque cas sans égaler les parents.

Ces observations se sont trouvées en concordance avec ce que les travaux de C. Daniel sur la génétique de l'Alisier torminal ont pu mettre en évidence à propos du degré de parenté de ces divers taxons.

\section{Intérêt syłvicole}

L'Alisier de Fontainebleau pourrait avoir un réel intérêt économique, dans certains milieux dont il supporterait mieux la contrainte édapho-climatique que le torminal, tout en ayant une meilleure conformation que le blanc.

II est dommage, Sorbus latifolia ètant actuellement une espèce protégée, que les forestiers ne soient pas encouragés à la protéger de la façon qui serait la plus simple et sûre, à savoir en la traitant et pérennisant comme il convient à une essence de production de valeur. $\dot{A}$ défaut, la rareté de cette belle espèce devrait quand même inciter à la faire profiter de dégagements, partout où la menacent la concurrence d'essences plus dynamiques et le vieillissement ou la conversion de peuplements. Car sans de telles mesures actives, la raréfaction de l'Alisier de Fontainebleau est certaine.

Sorbus confusa mérite également qu'on s'intéresse à lui. Les beaux spécimens n'en sont pas rares, qui accréditent fortement l'hypothèse de sa vigueur hybride; à tout le moins, ce serait aussi un taxon rustique et de meilleure conformation que l'Alisier blanc.

\section{AUTRES TAXONS}

Nous ne les citons que pour mémoire, étant donné leur intérêt forestier à peu près inexistant: ce sont soit des arbustes, soit des hybrides rares (donc difficiles à propager et méritant plutôt d'être protégés) ; certains de ceux-ci, pourtant, pourraient peut-être au moins égaler en taille le Sorbier des Oiseleurs.

Sorbus mougeotii forme de petits arbres ou des buissons, de l'étage montagnard moyen (surtout dans des milieux ouverts tels que les prés-bois) aux landes ou rochers subalpins.

Sorbus chamaemespilus, supportant des conditions encore plus rigoureuses, mais encore moins la concurrence, atteint les altitudes forestières supérieures (étage subalpin). En cohérence avec ces caractéristiques écologiques, cet Alisier n'est fondamentalement qu'un arbrisseau.

À l'instar du Sorbier des Oiseleurs en montagne, ces deux espèces apparaissent comme indifférentes au substrat dans les divers milieux où leur rusticité vis-à-vis des contraintes climatiques les rend concurrentielles.

\section{CONCLUSIONS}

\section{Écologie et éléments de sylviculture des Sorbus: synthèse}

Les Sorbus sont des plantes rustiques et à large amplitude écologique. Leur principale exigence concerne la lumière: ces arbres sont héliophiles, de tempérament dynamique post-pionnier nomade. Aussi sont-ils rares ou absents de milieux fertiles dans lesquels ils auraient de bonnes potentialités de croissance, mais ont à subir une trop forte concurrence interspécifique. 
Corollaire de cette sensibilité à la concurrence, l'abondance (et l'existence même) des Sorbus dans les peuplements est étroitement dépendante de la sylviculture. Leur sont favorables les structures plus ou moins dégradées, basses et claires, que la cause en soit stationnelle ou anthropique. Le traitement en taillis-sous-futaie a longtemps été profitable aux Sorbus, grâce à une hétérogénéité de structure qui a largement facilité la mise en réserve de ces "fruitiers " pour leur bois ou leur intérêt cynégétique (encore que cela dépende des espèces et d'habitudes parfois très locales). En revanche, la futaie régulière, donnant des peuplements toujours plus denses et/ou hauts, est très défavorable aux Sorbus en l'absence, comme cela a presque toujours été le cas jusqu'à maintenant, d'égards particuliers et suivis. De même l'abondance du Hêtre est cause d'une densité de couvert souvent fatale à ces arbres.

En dépit de fructifications souvent abondantes, c'est surtout par voie végétative que les Sorbus se régénèrent (drageonnement). Les fruits servent à la dissémination, grâce aux oiseaux.

\section{Perspectives}

L'intérêt de l'Alisier torminal n'est plus à démontrer, celui du Cormier largement admis... Bien des forestiers en auront au moins été convaincus par les cours de leurs bois. Mais il faut encore que les praticiens se convainquent de plusieurs choses:

- l'intérêt réel et complémentaire d'autres espèces de Sorbus et, pour toutes, que celui-ci s'étend à bien d'autres milieux que ceux où l'on peut déjà être accoutumé à les voir. Ne pas perdre de vue que les potentialités intrinsèques de ces essences sont souvent masquées par la concurrence (surtout pour le Cormier), et qu'on aurait tort de les réserver à des milieux ingrats ;

- la nécessité d'assurer à ces arbres une conduite attentionnée, d'appliquer une sylviculture intensive à peu d'individus plutôt qu'extensive à un grand nombre (option que doivent également faire privilégier les risques phytosanitaires et le coût des plantations). Si dans bien des situations les Sorbus "poussent tout seuls", c'est autre chose d'obtenir de beaux arbres ;

- l'intérêt d'expérimentations pratiques, qui sont largement du ressort des forestiers (plantations en conditions limites ou inédites, comparaison d'essences, essais de grands et petits plants, protection, fertilisation...).

Enfin, pour étendre le bénéfice de l'étude de l'Alisier torminal... et des autres Sorbus, est-il nécessaire d'attendre l'apparition de nouvelles modes pour commencer à plus souvent réserver des Érables champêtres ("il n'y a pas de marché " - mais voir dans la littérature les qualités de ce bois), des Bouleaux en milieux difficiles, des Tilleuls, peut-être même du Charme?

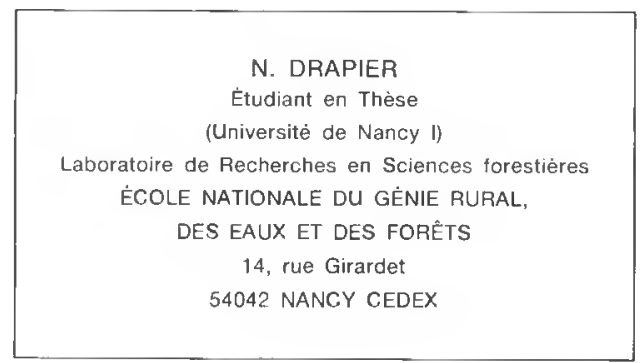

\section{BIBLIOGRAPHIE}

Voir aussi la bibliographie de l'article: "Écologie de l'Alisier torminal ", p. 229.

BECKER (M.), LE TACON (F.). - Santé de la forêt : importance d'une sylviculture adaptée aux conditions de milieu. - Revue forestière française, vol. XXXVII, $n^{\circ}$ spécial "Regards sur la santé de nos forêts ", 1985, pp. 7-28. 


\section{N. DRAPIER}

BOURGERY (C.), CASTANER (D.). - Les Plantations d'alignement, le long des routes, chemins, canaux, allées. - Paris : Institut pour le Développement forestier, 1988. - $416 \mathrm{p}$.

DANIEL (C.). - Étude de la diversité génétique de diverses provenances de Sorbus torminalis (L.) Crantz. Nancy: Université de Nancy I, 1991. - 64 p. + annexes (rapport de stage pour l'obtention du DEA de Biologie végétale et forestière).

DEMOLIS (C.). - Dépérissement de l'Alisier torminal (suite). - Arborescences, $n^{\circ} 21,1989$, pp. 6-7.

DRAPIER (N.). - À propos de la protection de l'Alisier de Fontainebleau. - Revue forestière française, vol. XLIII, $n^{\circ} 6,1991$, pp. 501-506.

FOURNIER (P.). - Le Livre des plantes médicinales et vénéneuses de France. — Tome 3. — Paris : Lechevalier, 1948. - $636 \mathrm{p}$.

HUBERT (M.). - Cultiver les arbres feuillus pour récolter des bois de qualité. - Paris: Institut pour le Développement forestier, 1981. - $277 \mathrm{p}$.

HUBERT (M.), COURRAUD (R.). - Élagage et taille de formation des arbres forestiers. - Paris : Institut pour le Développement forestier, 1987. - $292 \mathrm{p}$.

INSTITUT POUR LE DÉVELOPPEMENT FORESTIER. - Les Terrains boisés, leur mise en valeur. - $2^{\mathrm{e}}$ édition revue el corrigèe. - Paris: Institut pour le Développement forestier, 1980. - VIII + 254 p.

JACAMON (M.). - Guide de Dendrologie. Arbres, arbustes et arbrisseaux des forêts françaises. - $3^{e}$ édition. - Nancy: École nationale du Génie rural, des Eaux et des Forêts, 1992. - XVIII-350 p.

JACOBÉE (F.). - Propoșition d'élargissement de la base génétique de la production de plants d'Alisier torminal. - Nancy: École nationale du Génie rural, des Eaux et des Forêts, 1990 (rapport de stage de première année de FIF).

KAUSCH-BLECKEN VON SCHMELING (W.). - Der Speierling. — Göttingen : Fachhochschule, 1992. - 224 p.

KRUSSMANN (G.). - La Pépinière. Nouvelle adaptation française de la $4^{e}$ édition allemande. - Paris : La Maison Rustique, 1981. - $382 \mathrm{p}$.

LANIER (L.) et coll.. - Précis de sylviculture. - Nancy: École nationale du Génie rural, des Eaux et des Forêts, 1986. - VIII-468 p.

PHIPPS (J.B.), ROBERTSON (K.R.), ROHRER (J.R.), SMITH (P.G.). - A Synopsis of Genera in Maloideae (Rosaceae). - Systematic Botany, vol. 16, $n^{\circ} 2,1991$, pp. 376-394. 